\title{
Drug-Induced Tubulointerstitial Nephritis
}

National Cancer Institute

\section{Source}

National Cancer Institute. Drug-Induced Tubulointerstitial Nephritis. NCI Thesaurus.

Code C123014.

Tubulointerstitial nephritis resulting from a drug exposure. 